\title{
Venous thromboembolism and prophylaxis therapy after elective spine surgery: a population-based study
}

\section{Thromboembolie veineuse et traitement prophylactique après une chirurgie non urgente du rachis : une étude basée sur la population}

\author{
Megan Fiasconaro, MS · Jashvant Poeran, MD, PhD • Jiabin Liu, MD, PhD • \\ Lauren A. Wilson, MPH · Stavros G. Memtsoudis, MD, PhD, MBA
}

Received: 14 July 2020/Revised: 9 September 2020/Accepted: 9 September 2020/Published online: 18 November 2020

(C) Canadian Anesthesiologists' Society 2020

\begin{abstract}
Purpose Currently, there is no generalized consensus regarding perioperative prophylaxis of venous thromboembolism (VTE) in patients undergoing spine surgery. In the absence of large-scale studies, we aimed to use national data to study the association between anticoagulant prophylaxis and VTE in spine surgical patients. Our secondary outcomes were hematoma and blood transfusion.

Methods We included anterior cervical discectomy and fusion $(A C D F)$ and posterior lumbar fusion (PLF) cases from 2006 to 2016 recorded in the Premier Healthcare database. Anticoagulant prophylaxis was categorized into aspirin, regular heparin, and low molecular weight heparin given on the day of surgery. Mixed-effects
\end{abstract}

\footnotetext{
M. Fiasconaro, MS · L. A. Wilson, MPH

Department of Anesthesiology, Critical Care, and Pain

Management, Hospital for Special Surgery, 535 East 70th Street,

New York, NY, USA

J. Poeran, MD, $\mathrm{PhD}$

Department of Population Health Science and Policy, Institute for Healthcare Delivery Science, Icahn School of Medicine at Mount Sinai, New York, NY, USA

Leni and Peter W. May Department of Orthopaedics, Icahn School of Medicine at Mount Sinai, New York, NY, USA

J. Liu, MD, PhD

Department of Anesthesiology, Critical Care, and Pain

Management, Hospital for Special Surgery, 535 East 70th Street,

New York, NY, USA
}

Department of Anesthesiology, Weill Cornell Medical College, New York, NY, USA models measured the association between anticoagulation categories and outcomes. Cohorts were adjusted to reduce the risk of "confounding by indication" and to distinguish between prophylactic and therapeutic use of anticoagulants. We report odds ratios (OR) and Bonferroni-corrected confidence intervals $(\mathrm{CI})$.

Results Among 83,839 individuals undergoing ACDF and PLF, 0.45\% ( $n=374)$ had a hematoma, $8.1 \%(n=6,769)$ received a blood transfusion, and $0.13 \%(n=113)$ experienced VTE. After adjustment for relevant covariates, prophylactic aspirin (OR, 1.48; CI, 1.17 to $1.86)$ and regular heparin $(O R, 2.01 ; C I, 1.81$ to 2.24) were associated with increased odds of blood transfusion. No detectable differences in the odds of hematoma or VTE were observed for any anticoagulant.

S. G. Memtsoudis, MD, PhD, MBA ( $\square)$

Department of Anesthesiology, Critical Care, and Pain

Management, Hospital for Special Surgery, 535 East 70th Street,

New York, NY, USA

e-mail: MemtsoudisS@hss.edu

Department of Anesthesiology, Weill Cornell Medical College, New York, NY, USA

Department of Healthcare Policy and Research, Weill Cornell Medical College, New York, NY, USA

Department of Anesthesiology, Perioperative Medicine and Intensive Care Medicine, Paracelsus Medical University, Salzburg, Austria 
Conclusion Although low molecular weight heparin was used much less frequently than regular heparin, it was associated with a lower incidence of transfusion compared with aspirin and regular heparin. All three anticoagulants were associated with similar incidence of VTE and hematoma. Varying subgroup-specific VTE risks may further inform future studies to identify patients expected to benefit the most from chemical thromboprophylaxis.

\section{Résumé}

Objectif À l'heure actuelle, il n'existe pas de consensus concernant la prophylaxie périopératoire en cas de thromboembolie veineuse (TEV) pour les patients subissant une chirurgie du rachis. En l'absence d'études de grande envergure, nous avons cherché à utiliser des données nationales afin d'étudier l'association entre l'anticoagulothérapie et la TEV chez les patients de chirurgie $d u$ rachis. Nos critères d'évaluation secondaires étaient la présence d'hématome et les transfusions sanguines.

Méthode Nous avons inclus les chirurgies de discectomie cervicale antérieure avec fusion (DCAF) et de fusion lombaire postérieure (FLP) réalisées entre 2006 et 2016 et enregistrées dans la base de données Premier Healthcare. L'anticoagulothérapie a été catégorisée en aspirine, héparine normale, et héparine de bas poids moléculaire, donnée le jour de la chirurgie. Les modèles à effets mixtes ont mesuré l'association entre les catégories d'anticoagulation et les critères d'évaluation. Les cohortes ont été ajustées afin de réduire le risque de "confusion par indication" et de distinguer une utilisation prophylactique d'une utilisation thérapeutique des anticoagulants. Nous rapportons les rapports de cotes $(R C)$ et les intervalles de confiance (IC) corrigés par Bonferroni.

Résultats Parmi les 83839 personnes ayant subi une DCAF ou une FLP $0,45 \%(n=374)$ ont développé un hématome, $8,1 \%(n=6769)$ ont reçu une transfusion sanguine et $0,13 \%(n=113)$ ont souffert d'une TEV. Après ajustement pour tenir compte des covariables pertinentes, l'aspirine prophylactique (RC, 1,48; IC, 1,17 à 1,86) et l'héparine normale ( $R C, 2,01 ; \mathrm{IC}, 1,81$ à 2,24) ont été associées à des probabilités accrues de transfusion sanguine. Aucune différence détectable dans les risques d'hématome ou de TEV n'a été observée, indépendamment de l'anticoagulant utilisé.

Conclusion Bien que l'héparine de bas poids moléculaire ait été utilisée bien moins fréquemment que l'héparine normale, elle était associée à une incidence plus faible de transfusion par rapport à l'aspirine et à l'héparine normale. Les trois anticoagulants ont été associés à une incidence comparable de TEV et d'hématome. Les variations en matière de risque de TEV spécifiques aux sous-groupes pourraient orienter les études futures afin de tenter d'identifier les patients qui pourraient bénéficier le plus d'une thromboprophylaxie pharmaceutique.

Keywords anticoagulants · prophylaxis · spine surgery · venous thromboembolism

Venous thromboembolism (VTE, including deep vein thrombosis [DVT] and pulmonary embolism [PE]) is among the most serious complications after surgery. Here, benefits of antithrombotic prophylaxis have to be weighed carefully against potential harms, mainly bleeding. Particularly in the context of spinal surgery, this potential harm may be substantial as epidural hematoma-with an estimated incidence of $<1 \%^{1,2}$ may lead to neurologic deficits. Nevertheless, the reported burden of VTE in spinal surgery varies widely $(0.3-31 \%)$. $^{3}$

Currently, no widely accepted specific protocol exists for VTE prophylaxis in patients undergoing spinal surgery. Widely practiced and accepted interventions include the use of intermittent pneumatic compression and early mobilization as they are associated with no additional bleeding risk. Nevertheless, prophylactic use of anticoagulant agents have not been widely adopted given the aforementioned risks of bleeding and epidural hematoma.

Among the few studies addressing harms and benefits of specific protocols, Tacconi et al. concluded that their protocol involving all 5,347 patients with weight-based administration of low molecular weight heparin 24-48 hr post-surgery was safe and effective with a negligible risk of postoperative bleeding. ${ }^{4}$ Similar findings were reported by Akeda et al. ${ }^{5}$

While valuable, such studies evaluating singleinstitutional practices are limited in their generalizability, and it has been noted that large studies are desired to further investigate the safety of anticoagulant and antiplatelet medications as chemoprophylaxis. ${ }^{6}$ As a result, the purpose of our study was to utilize a national database to evaluate the risk of VTE for patients receiving various postoperative anticoagulant prophylaxis approaches (i.e., heparin, aspirin, warfarin) in the context of spinal surgery. Secondary outcomes of interests were hematoma and blood transfusion (as a marker of blood loss). We hypothesized that the risk of VTE would be low and that the odds for a bleeding-related complication in the setting of anticoagulant use would be manageable. 


\section{Methods}

Study design and sample

Patient information used in this retrospective cohort study was de-identified and obtained through the Premier Healthcare database (Premier Healthcare Solutions, Inc., Charlotte, NC, USA), which features patient information from electronic medical records and insurance claims. It comprises over 71 million visits per year since 2012, and represents approximately $20 \%$ of all annual inpatient discharges in the United States. The majority of hospitals captured within the Premier Healthcare database are located in the South, but the rest are evenly dispersed throughout the United States. ${ }^{7}$ International Classification of Diseases 9th revision (ICD-9) procedure codes were used to identify patients who underwent anterior cervical discectomy and fusion (ACDF; 81.02) or posterior lumbar fusion (PLF; 81.08) procedures from 2006 to 2016. Approval for this study was granted by the institutional review board (IRB\#2012-050) and the study was considered exempt from the requirement for informed consent.

We excluded entries with non-elective procedures, with unknown sex, with unknown discharge status, with outpatient procedures, treated at a hospital performing fewer than 30 procedures, or above the 95th percentile for total opioid utilization. We elected to exclude patients who received large quantities of opioids to make the study cohort more homogeneous. Spine patients with high opioid requirement are typically non-standard patients with significant comorbidity, prior surgeries, etc. (Figure). ${ }^{8,9}$

Study variables

The main outcomes of interest were hematoma, blood transfusion need (as a marker for blood loss), and VTE (DVT ICD-9 codes 451.1, 451.2, 451.8, 451.9, 453.2, 453.8, 453.9, 453.4; PE ICD-9 codes 415.11, 415.12, 415.19). The main effect of interest was the type of anticoagulant used; this included aspirin, regular heparin, low molecular weight heparin, and warfarin used on the day of surgery (day 0 ). The definition of anticoagulant use
Figure Identification of study cohort
Patients who had elective anterior cervical discectomy and fusion (ACDF; ICD-9 procedure code 81.02 ) or posterior lumbar fusion procedures (PLF; ICD-9 procedure code 81.08$)$ from $2006-2016(n=388,242)$

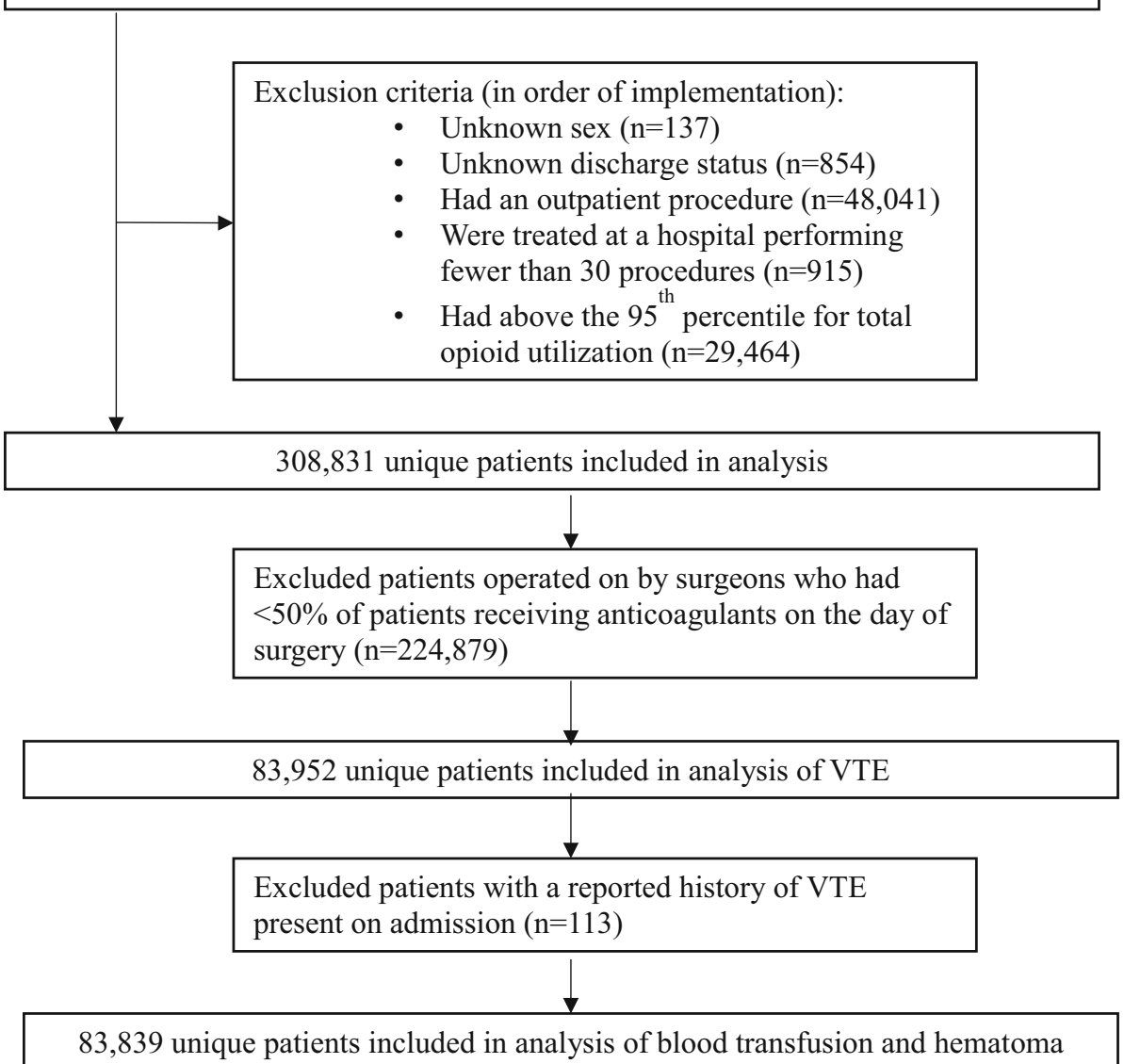


was restricted to use on the day of surgery to minimize the potential of misclassification, i.e., to distinguish between prophylactic and therapeutic use. Other study variables included demographic information such as age, sex, race (White, Black, and other), type of insurance (commercial, Medicaid, Medicare, uninsured, and other), and DeyoCharlson comorbidity index category. Hospital-related variables included hospital location (urban, rural), teaching status, hospital size (large, medium, small, in terms of beds), and hospital-specific procedure volume. Procedure-level variables were year of procedure, number of vertebrae fused ("low" as $\leq 3$, "high" as $>3$, or unknown), surgeon specialty (neurosurgery or orthopedic surgery), and opioid use during the entire hospitalization (high, medium, or low based on the interquartile range in oral morphine equivalents used).

\section{Statistical analysis}

Summary statistics were obtained for the outcomes of hematoma and blood transfusion by the study variables. Frequencies and percentages were reported for categorical variables. Standardized differences were applied to assess group differences in place of $t$ tests and rank sum tests as the large sample size of our study could cause the respective tests to be over-powered. ${ }^{10} \mathrm{~A}$ standardized difference greater than $10 \%(0.1)$ was used to indicate a meaningful difference in covariate distribution between the groups with and without the outcome. ${ }^{11}$

We applied a mixed-effects multivariable logistic regression model with a random intercept to account for correlation among patients treated at the same hospital (adjusting for all available covariates) to measure the association between the types of anticoagulant used (aspirin, regular heparin, low molecular weight heparin) and outcomes. Note that warfarin was not modelled given the observed low prevalence of use. Because some patients received more than one type of anticoagulant, we treated each of the three anticoagulants of interest as binary variables in multivariable models. Given the potential for confounding by indication, i.e., anticoagulant use in patients perceived at higher risk of VTE and the fact that our data set does not distinguish between prophylactic and therapeutic use of anticoagulants, we applied different cohort definitions when modelling 1) hematoma and blood transfusion need and 2) VTE. To reduce confounding by indication, we restricted the cohort to patients operated on by surgeons who had $>50 \%$ of their patients receiving anticoagulants on the day of surgery with the assumption that these surgeons practice under certain protocolized thromboprophylaxis plans. In scenarios where the majority of a surgeon's patients are receiving anticoagulants, it is more likely to be for prophylactic purposes than to treat a pre-existing condition. This cohort was applied to model VTE. In addition, we further restricted the study cohort by removing patients who had a diagnosis code reported for VTE paired with a present on admission indicator (thus indicating a preoperative history of VTE), to additionally ensure only prophylactic use of anticoagulants. This cohort was applied to model hematoma and blood transfusion need.

We report odds ratios (OR), confidence intervals (CI), and model c-statistics. Model c-statistics were calculated to evaluate model performance, a c-statistic of 0.7 or greater typically indiciates sufficient model discrimination. We applied a Bonferroni correction to adjust for the three studied outcomes and three comparisons (nine hypotheses testes); as a result, statistical significance was defined by a $P<0.006$. Analyses were conducted using SAS version 9.4 (SAS Institute, Cary, NC, USA). PROC GLIMMIX, which calculates robust standard errors, was used for modelling.

\section{Results}

We identified 308,831 individuals who underwent PLF or ACDF surgery from 2006 to 2016. Restricting the study cohort to cases from surgeons that we defined as using protocolized thromboprophylaxis yielded a study cohort of 83,839 individuals. Of these patients, $1,872(2.23 \%)$ were treated with only aspirin, $26,758(31.90 \%)$ were treated with only regular heparin, $888(1.06 \%)$ were treated with only low molecular weight heparin, $137(0.16 \%)$ were treated with only warfarin, and $942(1.12 \%)$ were treated with more than one anticoagulant.

We identified $0.45 \%(n=374)$ individuals in our cohort who experienced a hematoma during their hospital admission. Higher incidences of hematoma formation were seen in those who were older, non-White, on Medicare, treated in a large hospital, having a PLF procedure, operated on by an orthopedic surgeon and on more levels, with deformity, and having a higher comorbidity index. In addition, the incidence decreased over time; all with standardized differences $>0.1$ (Table 1) After adjusting for relevant covariates, there was no detectable difference between anticoagulant use and hematoma formation (Table 2).

There were $8.10 \%(n=6,769)$ cases that received a blood transfusion. Higher blood transfusion risks were seen in patients who were older, female, had Medicare insurance, had PLF surgery, were operated on by an orthopedic surgeon and with increasing levels of fusion, had a deformity, and had a higher comorbidity burden; all with standardized differences $>0.1$ (Table 1). Frequency of transfusions decreased over time. Overall, warfarin use was too infrequent to be included in our multivariable 
Table 1 Hematoma and blood transfusion need by study variables (cohort restricted to surgeons with $>50 \%$ use of prophylactic anticoagulation and exclusion of VTE; $n=83,839$ )

\begin{tabular}{|c|c|c|c|c|}
\hline & \multicolumn{2}{|l|}{ Hematoma } & \multicolumn{2}{|l|}{ Blood transfusion } \\
\hline & $(n=374)$ & STD & $(n=6,769)$ & STD \\
\hline Age* & $62(52,71)$ & 0.347 & $64(55,73)$ & 0.511 \\
\hline Length of stay* & $4(3,7)$ & 0.940 & $4(3,6)$ & 1.072 \\
\hline Sex: Female & $190(0.41 \%)$ & -0.094 & $4,484(9.65 \%)$ & 0.242 \\
\hline \multicolumn{5}{|l|}{ Race } \\
\hline Black & $50(0.63 \%)$ & 0.230 & $606(7.61 \%)$ & 0.091 \\
\hline Other & $67(0.66 \%)$ & & $985(9.64 \%)$ & \\
\hline White & $257(0.39 \%)$ & & $5,178(7.89 \%)$ & \\
\hline Discharge year & & 0.292 & & 0.573 \\
\hline 2006 & $45(0.6 \%)$ & & $954(12.73 \%)$ & \\
\hline 2007 & $43(0.53 \%)$ & & $926(11.48 \%)$ & \\
\hline 2008 & $62(0.77 \%)$ & & $974(12.05 \%)$ & \\
\hline 2009 & $39(0.37 \%)$ & & $1270(12.09 \%)$ & \\
\hline 2010 & $45(0.43 \%)$ & & $975(9.36 \%)$ & \\
\hline 2011 & $25(0.38 \%)$ & & $332(5 \%)$ & \\
\hline 2012 & $20(0.28 \%)$ & & $333(4.73 \%)$ & \\
\hline 2013 & $31(0.41 \%)$ & & $393(5.25 \%)$ & \\
\hline 2014 & $24(0.38 \%)$ & & $245(3.88 \%)$ & \\
\hline 2015 & $23(0.35 \%)$ & & $236(3.56 \%)$ & \\
\hline 2016 & $17(0.33 \%)$ & & $131(2.54 \%)$ & \\
\hline \multicolumn{5}{|l|}{ Insurance } \\
\hline Commercial & $156(0.41 \%)$ & 0.175 & $2,201(5.74 \%)$ & 0.389 \\
\hline Medicaid & $16(0.42 \%)$ & & $208(5.4 \%)$ & \\
\hline Medicare & $165(0.54 \%)$ & & $3,531(11.54 \%)$ & \\
\hline Uninsured & $2(0.33 \%)$ & & $29(4.79 \%)$ & \\
\hline Unknown & $35(0.34 \%)$ & & $800(7.68 \%)$ & \\
\hline \multicolumn{5}{|l|}{ Hospital size } \\
\hline Large & $167(0.49 \%)$ & 0.103 & $2,710(7.99 \%)$ & 0.028 \\
\hline Medium & $139(0.4 \%)$ & & $2,697(7.85 \%)$ & \\
\hline Small & $68(0.44 \%)$ & & $1,362(8.75 \%)$ & \\
\hline Teaching hospital & $193(0.42 \%)$ & -0.071 & $3,623(7.84 \%)$ & -0.035 \\
\hline Urban location & $361(0.45 \%)$ & -0.009 & $6,415(7.94 \%)$ & 0.085 \\
\hline \multicolumn{5}{|l|}{ Procedure } \\
\hline$A C D F$ & $190(0.39 \%)$ & 0.151 & $469(0.96 \%)$ & 1.446 \\
\hline$P L F$ & $184(0.53 \%)$ & & $6,300(18.01 \%)$ & \\
\hline \multicolumn{5}{|l|}{ Surgeon specialty } \\
\hline Neurosurgery & $43(0.38 \%)$ & 0.119 & $1,517(13.31 \%)$ & 1.032 \\
\hline Orthopedic surgery & $96(0.53 \%)$ & & $3,753(20.65 \%)$ & \\
\hline Unknown & $235(0.43 \%)$ & & $1,499(2.76 \%)$ & \\
\hline Hospital-specific annual surgery volume* & $409(249,692)$ & 0.016 & $400(226,694)$ & -0.057 \\
\hline \multicolumn{5}{|l|}{ Fusion level $($ low $<3$, high $\geq 4)$} \\
\hline High & $77(0.81 \%)$ & 0.287 & $1287(13.48 \%)$ & 0.270 \\
\hline Low & $284(0.41 \%)$ & & $5,334(7.61 \%)$ & \\
\hline Unknown & $13(0.31 \%)$ & & $148(3.49 \%)$ & \\
\hline Trauma & $3(0.79 \%)$ & 0.044 & $54(14.17 \%)$ & 0.048 \\
\hline Deformity & $46(1.02 \%)$ & 0.248 & $970(21.59 \%)$ & 0.338 \\
\hline Cancer & $6(0.65 \%)$ & 0.043 & $116(12.51 \%)$ & 0.057 \\
\hline
\end{tabular}


Table 1 continued

\begin{tabular}{|c|c|c|c|c|}
\hline & \multicolumn{2}{|l|}{ Hematoma } & \multicolumn{2}{|c|}{ Blood transfusion } \\
\hline & $(n=374)$ & STD & $(n=6,769)$ & STD \\
\hline Discharge status & & 0.545 & & 0.715 \\
\hline Dead & $3(8.57 \%)$ & & $9(25.71 \%)$ & \\
\hline Facility transfer & $92(1.39 \%)$ & & $1829(27.58 \%)$ & \\
\hline Home & $224(0.33 \%)$ & & $3,660(5.33 \%)$ & \\
\hline Home with help/other & $55(0.64 \%)$ & & $1271(14.88 \%)$ & \\
\hline Deyo-Charlson Index category & & 0.407 & & 0.224 \\
\hline 0 & $179(0.34 \%)$ & & $3,635(6.92 \%)$ & \\
\hline 1 & $112(0.52 \%)$ & & $1,887(8.72 \%)$ & \\
\hline 2 & $39(0.61 \%)$ & & $717(11.13 \%)$ & \\
\hline $3+$ & $44(1.34 \%)$ & & $530(16.19 \%)$ & \\
\hline Total opioid use & & 0.425 & & 0.512 \\
\hline $\operatorname{High}(>P 75)$ & $188(0.75 \%)$ & & $3,425(13.69 \%)$ & \\
\hline Medium (P25-P75) & $147(0.33 \%)$ & & $2,786(6.26 \%)$ & \\
\hline Low $(<P 25)$ & $39(0.27 \%)$ & & $558(3.9 \%)$ & \\
\hline \multicolumn{5}{|l|}{ Treated with one anticoagulant } \\
\hline Aspirin & $5(0.27 \%)$ & -0.068 & $133(7.10 \%)$ & -0.020 \\
\hline Regular heparin & $134(0.50 \%)$ & 0.083 & $4679(17.49 \%)$ & 0.886 \\
\hline LMWH & $8(0.88 \%)$ & 0.087 & $80(9.01 \%)$ & 0.013 \\
\hline Warfarin & $1(0.73 \%)$ & 0.023 & $12(8.76 \%)$ & 0.004 \\
\hline \multicolumn{5}{|c|}{ Treated with more than one anticoagulant } \\
\hline Aspirin + regular heparin & $1(0.15 \%)$ & -0.074 & $142(20.97 \%)$ & 0.120 \\
\hline Aspirin + LMWH & $0(0 \%)$ & -0.032 & $5(11.63 \%)$ & 0.010 \\
\hline Aspirin + warfarin & $0(0 \%)$ & -0.018 & $1(7.14 \%)$ & -0.002 \\
\hline Regular heparin + LMWH & $1(0.73 \%)$ & 0.023 & $26(18.98 \%)$ & 0.047 \\
\hline Regular heparin + warfarin & $0(0 \%)$ & -0.042 & $15(20.0 \%)$ & 0.037 \\
\hline LMWH + warfarin & $0(0 \%)$ & -0.022 & $2(10.00 \%)$ & 0.004 \\
\hline
\end{tabular}

*Median [interquartile range] for continuous variables. ACDF = anterior cervical discectomy and fusion; LMWH = low molecular weight heparin; STD = standardized difference; VTE = venous thromboembolism

analysis. Upon adjusting for covariates, the use of prophylactic aspirin (OR, 1.48; $\mathrm{CI}, 1.17$ to 1.86$)$ and regular heparin (OR, 2.01; $\mathrm{CI}, 1.81$ to 2.24$)$ were associated with an increased odds of blood transfusion need (Table 2).

Overall, the incidence of VTE was $0.13 \%$ ( $n=113$, of which 76 were DVT and 43 were PE). Higher VTE rates were found for patients who were older, female, nonWhite, had Medicare insurance, had surgery at large, urban and teaching hospitals, were undergoing PLF procedures with an increased number of levels fused, were undergoing surgery for deformity surgery, and had cancer. Additionally, a higher proportion of these cases were found across the group receiving anticoagulant therapy; all with standardized differences $>0.1$ (Table 3).

After adjusting for relevant covariates, we were unable to detect a difference in the odds of VTE for any of the three anticoagulants (Table 4). All multivariable models had c-statistics of 0.8 or greater, which indicates adequate model fit.

\section{Discussion}

In a cohort of 308,831 individuals undergoing ACDF and PLF in 478 United States hospitals, we identified 83,839 
Table 2 Mixed-effects multivariable model measuring the association between anticoagulant categories and hematoma and blood transfusion need (cohort restricted to surgeons with $>50 \%$ use of prophylactic anticoagulation and exclusion of VTE)

\begin{tabular}{|c|c|c|c|c|}
\hline & \multicolumn{2}{|c|}{ Outcome $=$ hematoma } & \multicolumn{2}{|c|}{ Outcome $=$ blood transfusion } \\
\hline & OR $(99.5 \% \mathrm{CI})$ & $P$ value & OR $(99.5 \% \mathrm{CI})$ & $P$ value \\
\hline Aspirin (reference: no aspirin) & $0.43(0.13$ to 1.39$)$ & 0.04 & $1.48(1.17$ to 1.86$)$ & $<0.001$ \\
\hline Regular heparin (reference: no heparin) & $0.85(0.58$ to 1.23$)$ & 0.21 & $2.01(1.81$ to 2.24$)$ & $<0.001$ \\
\hline LMWH (reference: no LMWH) & $\begin{array}{l}1.50(0.55 \text { to } 4.10) \\
\text { c-statistic }=0.87\end{array}$ & 0.26 & $\begin{array}{l}0.95(0.65 \text { to } 1.39) \\
\text { c-statistic }=0.91\end{array}$ & 0.70 \\
\hline
\end{tabular}

Models adjusted for age, sex, race, discharge year, insurance type, hospital bed size, teaching status and location, type of procedure, surgeon specialty, hospital-specific surgery volume, number of levels fused, trauma, deformity, cancer, Deyo-Charlson comorbidity index, and opioid use during hospitalization; full model results reported in Appendix 1

$\mathrm{CI}=$ confidence interval; $\mathrm{OR}=$ odds ratio; $\mathrm{LMWH}=$ low molecular weight heparin; $\mathrm{VTE}=$ venous thromboembolism.CI $=$ confidence interval; $\mathrm{OR}=$ odds ratio; $\mathrm{LMWH}=$ low molecular weight heparin; VTE = venous thromboembolism

cases that we considered to have received pharmacologic antithrombotic prophylaxis. Within this cohort, hematoma incidence was $0.45 \%$, which is in congruence with past literature where it is estimated to be $<1 \%$. $^{1,2}$ We found that, overall, prophylactic aspirin and regular heparin use were associated with an increase in odds of blood transfusion in the perioperative period after adjusting for relevant covariates. Nevertheless, anticoagulants were not associated with increased odds of hematoma formation. Overall, low molecular weight heparin appears to have the best benefit:harm profile given that we were unable to detect a difference in the odds of hematoma formation and blood transfusion or in the odds of VTE (there was no detectable difference in the odds of VTE for the other two anticoagulants as well). Since we were unable to detect a difference in the odds of these outcomes, the true relationship between these anticoagulants and VTE remains uncertain. Within this cohort, we did not find evidence to suggest an effect on risk of VTE; however, as no causal relationships can be established based on our data, it is possible that higher risk patients received these agents.

In our study considering aspirin, regular heparin, and low molecular weight heparin, we have found evidence that low molecular weight heparin may be associated with a better safety profile for the outcomes hematoma and blood transfusion. None of the pharmacologic agents were associated with hematoma formation; low molecular weight heparin was the only one that was not associated with an increased odds of blood transfusion, whereas aspirin and regular heparin were (OR, 1.48; CI, 1.17 to 1.86; and OR, 2.01; CI, 1.81 to 2.24 , respectively). In previous single-institutional prospective studies conducted by Tacconi et al. and Akeda et al., both used low molecular weight heparins and concluded that their protocols were safe, with insignificant risk of postoperative bleeding in the spine surgery population. ${ }^{4,5}$ While our results seem to confirm this finding in larger populations, it would be of interest for future research to compare effectiveness with other pharmacologic prophylaxis agents that are coming on the market at increasing rates.

In previous studies, identified risk factors for VTE include surgery type, comorbidities, prolonged surgery, deformity correction, and various hospital-related variables such as hospital location and size. ${ }^{12-14}$ Our study found univariable associations with many of our study variables, and confirmed significant associations with location, procedure type, deformity correction, cancer, and comorbidity burden as well. This shows that particular groups are at a higher risk of VTE, which should be taken into account when making harm $v s$ benefit evaluations for the administration of thromboprophylaxis in patients undergoing spine surgery. Indeed, more studies are needed that not only weigh benefits against harms in the 
Table 3 Venous thromboembolism by study variables (cohort restricted to surgeons with $>50 \%$ use of prophylactic anticoagulation; $n=83,952$ )

\begin{tabular}{|c|c|c|}
\hline & VTE $(n=113)$ & STD \\
\hline Age* & 67 [59-74] & 0.7 \\
\hline Length of stay* & 7 [5-9] & 1.3 \\
\hline Sex: female & $65(0.14 \%)$ & -1.5 \\
\hline \multicolumn{3}{|l|}{ Race } \\
\hline Black & $13(0.16 \%)$ & 0.7 \\
\hline Other & $18(0.18 \%)$ & \\
\hline White & $82(0.12 \%)$ & \\
\hline \multicolumn{3}{|l|}{ Discharge year } \\
\hline 2006 & $16(0.21 \%)$ & 6.10 \\
\hline 2007 & $22(0.27 \%)$ & \\
\hline 2008 & $12(0.15 \%)$ & \\
\hline 2009 & $13(0.12 \%)$ & \\
\hline 2010 & $16(0.15 \%)$ & \\
\hline 2011 & $7(0.11 \%)$ & \\
\hline 2012 & $8(0.11 \%)$ & \\
\hline 2013 & $6(0.08 \%)$ & \\
\hline 2014 & $3(0.05 \%)$ & \\
\hline 2015 & $5(0.08 \%)$ & \\
\hline 2016 & $5(0.10 \%)$ & \\
\hline \multicolumn{3}{|l|}{ Insurance } \\
\hline Commercial & $28(0.07 \%)$ & 4.023 \\
\hline Medicaid & $3(0.08 \%)$ & \\
\hline Medicare & $75(0.24 \%)$ & \\
\hline Uninsured & $0(0 \%)$ & \\
\hline Unknown & $7(0.07 \%)$ & \\
\hline \multicolumn{3}{|l|}{ Hospital size } \\
\hline Large & $53(0.16 \%)$ & 2.92 \\
\hline Medium & $45(0.13 \%)$ & \\
\hline Small & $15(0.10 \%)$ & \\
\hline Teaching hospital & $58(0.15 \%)$ & -1.568 \\
\hline Urban location & $113(0.14 \%)$ & -0.275 \\
\hline \multicolumn{3}{|l|}{ Procedure } \\
\hline$A C D F$ & $29(0.06 \%)$ & -1.19 \\
\hline$P L F$ & $84(0.24 \%)$ & \\
\hline \multicolumn{3}{|l|}{ Surgeon specialty } \\
\hline Neurosurgery & $32(0.28 \%)$ & 1.06 \\
\hline Orthopedic surgery & $36(0.20 \%)$ & \\
\hline Unknown & $45(0.08 \%)$ & \\
\hline Hospital-specific annual surgery volume* & $409[269-674]$ & -0.004 \\
\hline \multicolumn{3}{|c|}{ Fusion level (low $<3$, high $\geq 4)$} \\
\hline High & $17(0.18 \%)$ & 6.16 \\
\hline Low & $90(0.13 \%)$ & \\
\hline Unknown & $6(0.14 \%)$ & \\
\hline Trauma & $1(0.26 \%)$ & -0.096 \\
\hline Deformity & $14(0.31 \%)$ & -0.337 \\
\hline Cancer & $02(0.22 \%)$ & -0.150 \\
\hline Discharge status & & \\
\hline
\end{tabular}

Table 3 continued

\begin{tabular}{llc}
\hline & VTE $(n=113)$ & STD \\
\hline Dead & $5(12.5 \%)$ & 4.243 \\
Facility transfer & $40(0.60 \%)$ & \\
Home & $42(0.06 \%)$ & \\
Home with help/other & $26(0.3 \%)$ &
\end{tabular}

Deyo-Charlson Index categoryDeyo-Charlson Index category

$\begin{array}{ll}0 & 44(0.08 \%) \\ 1 & 40(0.18 \%) \\ 2 & 17(0.26 \%) \\ 3+ & 12(0.37 \%)\end{array}$

Total opioid use

$\begin{array}{ll}\text { High }(>P 75) & 59(0.24 \%) \\ \text { Medium }(P 25-P 75) & 39(0.09 \%) \\ \text { Low }(<P 25) & 15(0.10 \%)\end{array}$

8.042

Treated with one anticoagulant

Aspirin

$4(0.21 \%) \quad-0.253$

Regular heparin

$54(0.20 \%) \quad-0.992$

LMWH

$0(0.0 \%)-0.161$

Warfarin

$1(0.72 \%) \quad 0.239$

Treated with more than one anticoagulant

$\begin{array}{llr}\text { Aspirin + regular heparin } & 1(0.15 \%) & 0.078 \\ \text { Aspirin + LMWH } & 1(2.27 \%) & 0.122 \\ \text { Aspirin + warfarin } & 0(0 \%) & -0.018 \\ \text { Regular Heparin + LMWH } & 1(0.72 \%) & 0.100 \\ \text { Regular Heparin + warfarin } & 0(0 \%) & -0.042 \\ \text { LMWH + warfarin } & 3(13.00 \%) & 0.230\end{array}$

*Median [interquartile range] for continuous variables. ACDF = anterior cervical discectomy and fusion; LMWH = low molecular weight heparin; PLF $=$ posterior lumbar fusion; STD $=$ standardized difference

total spinal surgery population but also identify those at higher baseline risk of VTE, including those with (history of) malignancies and those with preoperative immobility. ${ }^{3}$

Limitations of this study include the retrospective design. We cannot make any statements on causality; only associations and risk factors were assessed. As a result of our data source, important clinical variables are missing, and errors may have occurred in data collection. Identifying procedure and complication variables through ICD-9 codes could have been limited by issues with coding accuracy and compliance. As a consequence of this, we were dependent on the number of vertebrae fused as our only measure of surgical complexity. There are other 
Table 4 Mixed-effects multivariable model measuring the association between anticoagulant categories and VTE (cohort restricted to surgeons with $>50 \%$ use of prophylactic anticoagulation)

\begin{tabular}{lll}
\hline & Outcome= VTE \\
\cline { 2 - 3 } & Odds ratio (99.5\% CI) & $P$ value \\
\hline Aspirin (reference: no aspirin) & $1.24(0.37$ to 4.11$)$ & 0.61 \\
Regular heparin (reference: no regular heparin) & $1.00(0.54$ to 1.85$)$ & 1.00 \\
LMWH (reference: no LMWH) & $2.41(0.61$ to 9.59$)$ & 0.07 \\
& c-statistic= 0.80 & 0 \\
\hline
\end{tabular}

Model adjusted for age, sex, race, discharge year, insurance type, hospital bed size, teaching status and location, type of procedure, surgeon specialty, hospital-specific surgery volume, number of levels fused, trauma, deformity, cancer, Deyo-Charlson comorbidity index, and opioid use during hospitalization; full model results reported in Appendix 2

$\mathrm{CI}=$ confidence interval; $\mathrm{LMWH}=$ low molecular weight heparin; VTE = venous thromboembolism

unmeasured factors that may confound the observed relationships; therefore, further study with more detailed information is needed to validate these findings. Similarly, mean blood loss might be more relevant than blood transfusion for our study purpose. Nevertheless, such information could not be extracted from the database, so we adopted blood transfusion as proxy. Additionally, because of the retrospective nature of the database, we were not able to formally distinguish between acute and chronic VTE. We attempted to control for this by excluding patients with a preoperative history of VTE, but were unable to assess the timing of VTE and cannot guarantee that all preoperative VTEs were recorded. Our study focused on postoperative day (POD) 0 VTE prophylaxis, while many institutions do not routinely start prophylaxis until later, such as POD 1. Such practice might not have been captured in our analysis. In addition, while we attempted to define anticoagulant prophylaxis utilizing the data available, there is no definitive way to conclude that use of anticoagulants on day 0 represents protocolized prophylactic interventions. We could not determine whether patients were treated with anticoagulants because they were high risk, potentially introducing selection bias. We attempted to control for this by only including surgeons with $>50 \%$ of their patients receiving anticoagulants on the day of surgery, which supports more of a prophylactic strategy. Further, the impact and utilization of more invasive interventions such as those involving inferior vena cava filters have not been considered. In addition to estimating the protocolization of anticoagulants in this study, we also acknowledge that there is an additional question of whether there is an effect on the timing of chemoprophylaxis for patients undergoing surgery. While no consensus has been reached to date, ${ }^{3}$ we did not have data available to distinguish the time between surgery and prophylaxis within the day of surgery. Furthermore, our study did not include all available regiments of VTE prophylaxis and the total number of patients who received VTE prophylaxis on POD 0 was low, therefore certain effects might not have been detected.

In summary, among patients undergoing ACDF and PLF surgery who were categorized as receiving antithrombotic prophylaxis, we found a significant increase in the odds of blood transfusions for those who received regular heparin or aspirin on the day of surgery. Although far fewer patients received low molecular weight heparin compared with regular heparin, no such association was seen with low molecular weight heparin. The three anticoagulant variables were not associated with a significant increase in the odds of hematoma formation. While there was no difference in experiencing VTE among those who received these agents compared with those who did not, this finding is potentially subject to indication bias, even after restricting our study cohort to minimize this. In addition, as we were able to identify some modifiable risk factors for VTE in our analysis, efforts may need to focus on risk stratification to identify those who might benefit most from pharmacologic interventions. It is possible that agents such as low molecular weight heparin, which had the lowest odds for adverse effects, are indicated in sub-populations such as those with advanced age and increased comorbidity burden.

Author contributions Megan Fiasconaro and Lauren A. Wilson contributed to all aspects of this manuscript, including study conception and design; acquisition, analysis, and interpretation of data; and drafting the article. Jashvant Poeran, Jiabin Liu, and Stavros G. Memtsoudis contributed to the conception and design of the study, acquisition and interpretation of data, and drafting the article. 
Disclosures None.

Funding statement The current study is funded by Department of Anesthesiology, Critical Care, and Pain Management, Hospital for Special Surgery, New York, NY.
Editorial responsibility This submission was handled by Dr. Alana M. Flexman, Associate Editor, Canadian Journal of Anesthesia.

\section{Appendix 1 Mixed-effects multivariable model measuring the association between anticoagulant categories and hematoma and blood transfusion need (cohort restricted to surgeons with $>50 \%$ use of prophylactic anticoagulation and exclusion of VTE)}

\begin{tabular}{|c|c|c|c|c|}
\hline \multirow[t]{2}{*}{ Characteristic } & \multicolumn{2}{|l|}{ Hematoma } & \multicolumn{2}{|l|}{ Blood transfusion } \\
\hline & OR $(99.5 \% \mathrm{CI})$ & $P$ value & OR $(99.5 \% \mathrm{CI})$ & $P$ value \\
\hline Age, continuous* & 1.03 (1.02 to 1.05$)$ & $<0.001$ & 1.03 (1.03 to 1.04$)$ & $<0.001$ \\
\hline Gender & & 0.02 & & $<0.001$ \\
\hline Female & 0.78 (0.58 to 1.05$)$ & & $1.61(1.47$ to 1.76$)$ & \\
\hline Male & Reference & & Reference & \\
\hline Race & & $<0.001$ & & $<0.001$ \\
\hline Black & 1.74 (1.11 to 2.72$)$ & & $1.27(1.09$, to 1.48$)$ & \\
\hline Other & 1.70 (1.13 to 2.54$)$ & & $1.02(0.87$ to 1.19$)$ & \\
\hline White & Reference & & Reference & \\
\hline Discharge year & & 0.003 & & $<0.001$ \\
\hline 2007 & $0.90(0.49$ to 1.64$)$ & & $0.81(0.69$ to 0.96$)$ & \\
\hline 2008 & $1.24(0.71$ to 2.18$)$ & & $0.84(0.71$ to 0.99$)$ & \\
\hline 2009 & $0.60(0.32$ to 1.13$)$ & & $0.81(0.69$ to 0.95$)$ & \\
\hline 2010 & 0.69 (0.37 to 1.28$)$ & & $0.62(0.52$ to 0.74$)$ & \\
\hline 2011 & 0.65 (0.31 to 1.35$)$ & & $0.60(0.47$ to 0.75$)$ & \\
\hline 2012 & $0.49(0.22$ to 1.06$)$ & & $0.62(0.49$ to 0.79$)$ & \\
\hline 2013 & 0.67 (0.34 to 1.33$)$ & & $0.52(0.41,0.65)$ & \\
\hline 2014 & $0.63(0.30$ to 1.31$)$ & & $0.34(0.26$ to 0.43$)$ & \\
\hline 2015 & $0.56(0.26$ to 1.20$)$ & & $0.32(0.24$ to 0.41$)$ & \\
\hline 2016 & $0.55(0.22$ to 1.39$)$ & & $0.00(0.00$ to 0.00$)$ & \\
\hline 2006 & Reference & & Reference & \\
\hline Insurance & & 0.28 & & 0.08 \\
\hline Medicaid & $1.02(0.48$ to 2.17$)$ & & $0.37(0.25$ to 0.53$)$ & \\
\hline Medicare & $0.74(0.49$ to 1.09$)$ & & $1.01(0.79$ to 1.30$)$ & \\
\hline Uninsured & $0.94(0.13$ to 7.00$)$ & & $1.06(0.95$ to 1.20$)$ & \\
\hline Unknown & $0.85(0.50$ to 1.45$)$ & & $1.29(0.69$ to 2.42$)$ & \\
\hline Commercial & Reference & & Reference & \\
\hline Hospital size & & 0.44 & & 0.95 \\
\hline Large & 0.97 (0.58 to 1.62$)$ & & $0.94(0.39$ to 2.28$)$ & \\
\hline Medium & 0.85 (0.55 to 1.32$)$ & & $0.93(0.46$ to 1.86$)$ & \\
\hline Small & Reference & & Reference & \\
\hline Teaching hospital & & 0.29 & & 0.65 \\
\hline Yes & $1.13(0.82$ to 1.57$)$ & & $0.91(0.49$ to 1.68$)$ & \\
\hline No & Reference & & Reference & \\
\hline Location & & 0.67 & & 0.46 \\
\hline Rural & 1.14 (0.48 to 2.68$)$ & & $1.38(0.41$ to 4.65$)$ & \\
\hline
\end{tabular}


Appendix continued

\begin{tabular}{|c|c|c|c|c|}
\hline \multirow[t]{2}{*}{ Characteristic } & \multicolumn{2}{|l|}{ Hematoma } & \multicolumn{2}{|l|}{ Blood transfusion } \\
\hline & OR $(99.5 \% \mathrm{CI})$ & $P$ value & OR $(99.5 \% \mathrm{CI})$ & $P$ value \\
\hline Urban & Reference & & Reference & \\
\hline Procedure & & 1.00 & & $<0.001$ \\
\hline PLF & $1.00(0.56$ to 1.80$)$ & & $17.56(14.08$ to 21.90$)$ & \\
\hline $\mathrm{ACDF}$ & Reference & & Reference & \\
\hline MD & & 0.18 & & 0.001 \\
\hline Neurosurgery & 0.84 (0.49 to 1.42$)$ & & $0.82(0.71$ to 0.95$)$ & \\
\hline Unknown & 1.27 (0.74 to 2.17$)$ & & $0.91(0.76$ to 1.08$)$ & \\
\hline Orthopedic surgery & Reference & & Reference & \\
\hline Provider volume* & $1.00(1.00$ to 1.00$)$ & 0.60 & $1.00(1.00$ to 1.00$)$ & 0.83 \\
\hline Fusion level (low $<3$, high $\geq 4)$ & & 0.02 & & $<0.001$ \\
\hline High & $1.47(1.00$ to 2.16$)$ & & 2.11 (1.86 to 2.39$)$ & \\
\hline Unknown & $0.94(0.37$ to 2.39$)$ & & $0.59(0.43,0.81)$ & \\
\hline Low & Reference & & Reference & \\
\hline Trauma & & 0.48 & & 0.10 \\
\hline No & $1.52(0.29$ to 7.98$)$ & & $1.35(0.81$ to 2.23$)$ & \\
\hline Yes & Reference & & Reference & \\
\hline Deformity & & 0.001 & & $<0.001$ \\
\hline No & 1.76 (1.08 to 2.84$)$ & & 1.58 (1.38 to 1.82$)$ & \\
\hline Yes & Reference & & Reference & \\
\hline Cancer & & 0.51 & & 0.14 \\
\hline No & $0.76(0.23$ to 2.51$)$ & & $1.21(0.85$ to 1.71$)$ & \\
\hline Yes & Reference & & Reference & \\
\hline Deyo-Charlson Index category & & $<0.001$ & & $<0.001$ \\
\hline 1 & $1.36(0.97$ to 1.93$)$ & & $1.12(1.01$ to 1.23$)$ & \\
\hline 2 & $1.52(0.91$ to 2.54$)$ & & $1.38(1.19$ to 1.60$)$ & \\
\hline $3+$ & $3.14(1.88$, to 5.25$)$ & & $2.19(1.82$ to 2.63$)$ & \\
\hline 0 & Reference & & Reference & \\
\hline Total opioid use & & $<.0001$ & & $<0.0001$ \\
\hline High & $3.25(1.91$ to 5.53$)$ & & $1.40(1.18$ to 1.66$)$ & \\
\hline Medium & $1.32(0.79$ to 2.20$)$ & & $1.05(0.89$ to 1.24$)$ & \\
\hline Low & Reference & & Reference & \\
\hline Day 0 aspirin & $0.43(0.13$ to 1.39$)$ & 0.04 & $1.48(1.17$ to 1.86$)$ & $<0.0001$ \\
\hline Day 0 regular heparin & $0.85(0.58$ to 1.23$)$ & 0.21 & 2.01 (1.81 to 2.24$)$ & $<0.0001$ \\
\hline Day 0 low molecular weight heparin & $1.50(0.55$ to 4.10$)$ & 0.26 & $0.95(0.65$ to 1.39$)$ & 0.70 \\
\hline & c-statistic $=0.87$ & & c-statistic $=0.91$ & \\
\hline
\end{tabular}

$\mathrm{ACDF}=$ anterior cervical discectomy and fusion; $\mathrm{CI}=$ confidence interval; $\mathrm{MD}=$ surgeon specialty; $\mathrm{OR}=$ odds ratio; $\mathrm{PLF}=$ posterior lumbar fusion; VTE = venous thromboembolism 


\section{Appendix 2 Mixed-effects multivariable model measuring the association between anticoagulant categories and VTE (cohort restricted to surgeons with $>50 \%$ use of prophylactic anticoagulation)}

\begin{tabular}{|c|c|c|}
\hline Characteristic & $\begin{array}{l}\text { VTE } \\
\text { OR }(99.5 \% \text { CI })\end{array}$ & $P$ value \\
\hline Age, continuous* & 1.05 (1.03 to 1.08$)$ & $<0.001$ \\
\hline Gender & & 0.80 \\
\hline Female & $0.95(0.56$ to 1.63$)$ & \\
\hline Male & Reference & \\
\hline Race & & 0.17 \\
\hline Black & 1.67 (0.71 to 3.92$)$ & \\
\hline Other & 1.33 (0.63 to 2.80$)$ & \\
\hline White & Reference & \\
\hline Discharge year & & 0.72 \\
\hline 2007 & $1.24(0.49$ to 3.15$)$ & \\
\hline 2008 & $0.67(0.23$ to 1.96$)$ & \\
\hline 2009 & $0.55(0.19$ to 1.59$)$ & \\
\hline 2010 & $0.68(0.25$ to 1.88$)$ & \\
\hline 2011 & $0.67(0.18$ to 2.50$)$ & \\
\hline 2012 & $0.66(0.19$ to 2.31$)$ & \\
\hline 2013 & $0.43(0.11$ to 1.69$)$ & \\
\hline 2014 & $0.24(0.04$ to 1.46$)$ & \\
\hline 2015 & $0.34(0.07$ to 1.54$)$ & \\
\hline 2016 & $0.38(0.07$ to 2.24$)$ & \\
\hline 2006 & Reference & \\
\hline \multicolumn{3}{|l|}{ Insurance } \\
\hline Medicaid & - & \\
\hline Medicare & - & \\
\hline Uninsured & - & \\
\hline Unknown & - & \\
\hline Commercial & - & \\
\hline Hospital size & & 0.72 \\
\hline Large & $1.31(0.49$ to 3.48$)$ & \\
\hline Medium & $1.13(0.48$ to 2.70$)$ & \\
\hline Small & Reference & \\
\hline Teaching hospital & & 0.49 \\
\hline Yes & 1.15 (0.65 to 2.04$)$ & \\
\hline No & Reference & \\
\hline \multicolumn{3}{|l|}{ Location } \\
\hline Rural & - & \\
\hline Urban & - & \\
\hline Procedure & & 0.09 \\
\hline PLF & $1.91(0.66$ to 5.51$)$ & \\
\hline $\mathrm{ACDF}$ & Reference & \\
\hline MD & & 0.12 \\
\hline Neuro & 1.65 (0.82 to 3.32$)$ & \\
\hline Unknown & $1.13(0.46$ to 2.75$)$ & \\
\hline Ortho & Reference & \\
\hline
\end{tabular}

Appendix continued

\begin{tabular}{lll}
\hline Characteristic & VTE & $P$ value \\
\hline OR $(99.5 \% \mathrm{CI})$ & \\
\hline Fusion level (low $<3$, high $\geq 4)$ & $1.00(1.00$ to 1.00$)$ & 0.99 \\
High & $1.05(0.48$ to 2.28$)$ & \\
Unknown & $1.52(0.33$ to 6.94$)$ & \\
Low & Reference &
\end{tabular}

Trauma

No

Yes

Deformity

No

Yes

Cancer

No

Yes

Deyo-Charlson Index category

1.42 (0.08 to 24.58$)$

Reference

1.39 (0.5 to, 3.26)

Reference

0.77 (0.10 to 6.00$)$

Reference

2

$3+$

0

Total opioid use

High

Medium

Low

Day 0 aspirin

Day 0 regular heparin

Day 0 Low molecular weight heparin 2.41 (0.61 to 9.59)

0.61

1.00 0.07

c-statistic $=0.80$

$\mathrm{ACDF}=$ anterior cervical discectomy and fusion; $\mathrm{CI}=$ confidence interval; $\mathrm{OR}=$ odds ratio; $\mathrm{MD}=$ surgeon specialty; $\mathrm{PLF}=$ posterior lumbar fusion; VTE = venous thromboembolism

\section{References}

1. Yi S, Yoon DH, Kim KN, Kim SH, Shin HC. Postoperative spinal epidural hematoma: risk factor and clinical outcome. Yonsei Med J 2006; 47: 326-32.

2. Glotzbecker MP, Bono CM, Wood KB, Harris MB. Thromboembolic disease in spinal surgery: a systematic review. Spine (Phila Pa 1976) 2009; 34: 291-303.

3. Kepler CK, McKenzie J, Kreitz T, Vaccaro A. Venous thromboembolism prophylaxis in spine surgery. J Am Acad Orthop Surg 2018; 26: 489-500.

4. Tacconi L, Spinelli R, Manganotti P. The prevention of deep venous thrombosis in neurosurgery: an update from our institution. EuroMediterranean Biomed J 2018; 13: 160-3.

5. Akeda $K$, Matsunaga $H$, Imanishi $T$, et al. Prevalence and countermeasures for venous thromboembolic diseases associated with spinal surgery: a follow-up study of an institutional protocol in 209 patients. Spine 2014; 39: 791-7. 
6. Alvarado AM, Porto GB, Wessell J, Buchholz AL, Arnold PM. Venous thromboprophylaxis in spine surgery. Global Spine $\mathbf{J}$ 2020; 10: 65S-70S.

7. Premier Applied Sciences ${ }^{\circledR ;}$ Premier Inc. Premier Healthcare Database: Data That Informs and Performs - March 2, 2020. Available from URL; https://products.premierinc.com/ downloads/PremierHealthcareDatabaseWhitepaper.pdf (accessed September 2020).

8. Armaghani SJ, Lee DS, Bible JE, et al. Preoperative opioid use and its association with perioperative opioid demand and postoperative opioid independence in patients undergoing spine surgery. Spine (Phila Pa 1976) 2014; 39: E1524-30.

9. Sanford Z, Broda A, Taylor H, Turcotte J, Patton CM. Predictive risk factors associated with increased opioid use among patients undergoing elective spine surgery. Int J Spine Surg 2020; 14: 189-94.

10. Austin PC. An introduction to propensity score methods for reducing the effects of confounding in observational studies. Multivariate Behav Res 2011; 46: 399-424.

11. Yang $D$, Dalton J. A unified approach to measuring the effect size between two groups using SAS ${ }^{\circledR}$. support.sas.com: SAS; 2012.
Available from URL: https://www.semanticscholar.org/paper/Aunified-approach-to-measuring-the-effect-size-two-Yang-Dalton/ 6cf4bd36ca4c90006a5d6563f646a391c255581b (accessed September 2020).

12. Park JH, Lee KE, Yu YM, Park YH, Choi SA. Incidence and risk factors for venous thromboembolism after spine surgery in Korean patients. World Neurosurg 2019; 128: e289-307.

13. Kim HJ, Iyer $S$, Diebo $B G$, et al. Clinically significant thromboembolic disease in adult spinal deformity surgery: incidence and risk factors in 737 patients. Global Spine J 2018; 8: 224-30.

14. Charen DA, Qian ET, Hutzler LH, Bosco JA. Risk factors for postoperative venous thromboembolism in orthopaedic spine surgery, hip arthroplasty, and knee arthroplasty patients. Bull Hosp Jt Dis 2013; 2015(73): 198-203.

Publisher's Note Springer Nature remains neutral with regard to jurisdictional claims in published maps and institutional affiliations. 